Published in final edited form as:

ACS Appl Mater Interfaces. 2019 April 03; 11(13): 12216-12223. doi:10.1021/acsami.8b19236.

\title{
Extended nitric oxide-releasing polyurethanes via S-nitrosothiol- modified mesoporous silica nanoparticles
}

\author{
Maggie J. Malone-Povolny, Mark H. Schoenfisch ${ }^{\star}$ \\ Department of Chemistry, University of North Carolina at Chapel Hill, CB3290, Chapel Hill, North \\ Carolina, 27599, United States
}

\begin{abstract}
$S$-nitrosothiol (RSNO)-modified mesoporous silica nanoparticles (MSNs) were doped into polyurethane (PU) to achieve extended NO-releasing coatings. Parameters influencing the synthesis of RSNO-functionalized nitric oxide (NO)-releasing MSNs were evaluated to elucidate the impact of pore structure on NO-release characteristics. The porous particles were characterized as having larger NO payloads and longer NO-release durations than that of nonporous particles, a feature attributed to recombination of the NO radical in confined intraporous microenvironments. Nitric oxide-release kinetics, particle leaching, and thermal stability of the RSNO-modified MSNs dispersed in PU were evaluated as a function of PU structure to determine the feasibility of preparing a range of NO-releasing polymers for biomedical device coating applications. The NOrelease kinetics from the PUs proved to be highly extended ( $>30 \mathrm{~d}$ ) and consistent over a range of polyurethane properties. Furthermore, RSNO-modified MSN leaching was not observed from the PUs. The NO-release payloads were also maintained for 4 days for polymers stored at $0{ }^{\circ} \mathrm{C}$.
\end{abstract}

\section{Graphical Abstract}

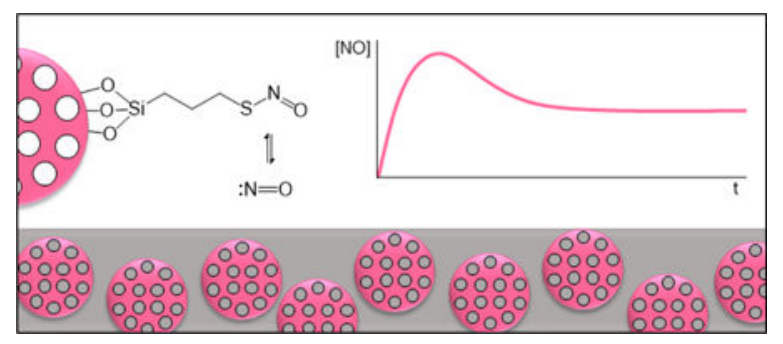

\section{Keywords}

nitric oxide; polyurethane; $S$-nitrosothiol; silica nanoparticle; mesoporosity

\footnotetext{
*Corresponding Author: schoenfisch@unc.edu. SUPPORTING INFORMATION.

NO-release from polyurethanes cast on glass; NO release of nonporous-particle doped polyurethane membranes; storage stability of nonporous particle-doped polyurethane membranes

The corresponding author declares the following competing financial interest(s): Mark Schoenfisch maintains financial interest in Clinical Sensors, Inc. and Vast Therapeutics, Inc. Clinical Sensors is developing NO-releasing sensors membranes for continuous glucose monitoring devices. Vast Therapeutics is commercializing NO-releasing macromolecular vehicles for dermatological indications.
} 


\section{INTRODUCTION}

Nitric oxide (NO), an endogenously produced free radical, plays a role in numerous physiological processes, including the inflammatory response, angiogenesis, vasodilation, and antimicrobial and tumoricidal activity. ${ }^{1-5}$ As a result, much research has focused on the potential benefits of exogenously supplied NO for therapeutic applications. The effects of NO are generally local due to its high reactivity and short lifetime in physiological milieu. ${ }^{6}$ Nitric oxide's role in wound healing has motivated the development of medical device polymeric coatings with NO-release capabilities to improve tissue integration and device performance. ${ }^{7-9}$ Common strategies for the active release of drugs from a medical device include outer coatings doped with traditional antibiotics to limit infection, ${ }^{10}$ dexamethasone to reduce inflammation, ${ }^{11}$ or gene therapeutic agents to stimulate vascular growth. ${ }^{12}$ Nitric oxide is unique in that it is capable of providing each of these benefits in a single drugrelease configuration. Development of a range of NO-releasing macromolecular scaffolds, including nanoparticles, ${ }^{13,14}$ polysaccharides, ${ }^{15,16}$ and dendrimers ${ }^{17}$ has provided NOrelease systems with a wide array of scaffold characteristics and release profiles to suit multiple medical applications.

Silica nanoparticles (SNPs) represent an attractive NO-releasing macromolecular scaffold for medical device coatings. Silica is bioinert, easily functionalizable, and can be readily encapsulated in the polymeric coatings commonly used in the design and fabrication of a given device. ${ }^{18-20}$ Preliminary studies using SNP-based NO-releasing intravascular catheter coatings and subcutaneous glucose sensor membranes have shown reduced thrombosis and tissue inflammation, respectively. ${ }^{21,22}$ The promising results from these studies prompted the development of more efficient and effective NO-delivery systems to achieve targeted, tunable, and extended delivery of NO from polymeric coatings. Extended release durations, in particular, have been implicated in better device outcomes: lower thrombosis, improved analyte uptake into microneedles, and decreased inflammatory tissue markers. ${ }^{22-24}$

Previous work related to NO-releasing SNP-doped coatings have primarily focused on $N$ diazeniumdiolate (NONOate)-modified SNPs. ${ }^{13,22,25}$ The decomposition rate of NONOates (and liberation of NO) relies on the uptake of water (protons) by the polymer, which imposes a restriction on the design of the system, wherein hydrophobic matrices are required to extend the release of NO. Such requirement is challenging for applications that favor more hydrophilic materials to prevent biofouling (e.g., wound dressings and implanted sensors). In contrast, the use of $S$-nitrosothiol (RSNO) NO donors does not impose the same design restrictions as NONOates, as the NO release from RSNOs is based on a photothermal decomposition mechanism, independent of water uptake or local $\mathrm{pH}$. While the lability of RSNOs species allows them to release NO spontaneously under physiological conditions, photothermal instability is detrimental with respect to storage of the material. Previous work has emphasized that RSNO-based scaffolds are susceptible to ambient light and heat, which poses a challenge for their translation to large-scale commercial distribution. ${ }^{26-28}$

Mesoporous silica nanoparticles (MSNs) are SNPs with an array of mesochannels that may address the stability challenges commonly associated with RSNO-modified SNPs. The inherently large interior surface area of MSNs may confer additional stability to intraporous 
RSNO groups due to a phenomenon known as the cage effect. First defined by Franck and Rabinowitch, ${ }^{29,30}$ the cage effect describes the reduction of radical species formation via geminate recombination within the confines of strong solvent "cages". These cages are formed by viscous solvents and/or local structural confinement. ${ }^{31,32}$ In the case of RSNOs, the cage effect favors recombination of the thiyl and NO radical pair after homolytic S-N bond cleavage, extending the duration of NO delivery. ${ }^{33,34}$ The confined microenvironment provided by the nanometer-scale pores of an MSN may reduce the rate of thermal and photochemical NO release in comparison to RSNO groups found on the exterior surface area of a particle.

Herein, we report on the synthesis and characterization of RSNO-functionalized MSNs with extended NO-release durations. The physiochemical properties of the MSNs are compared to nonporous analogues to elucidate the impact of pores on the NO-release properties of the SNP scaffold. In addition, we describe the encapsulation of the RSNO-functionalized MSNs in a range of polyurethane coatings to evaluate the role of polymer hydrophobicity on NOrelease kinetics, particle leaching, and stability.

\section{EXPERIMENTAL}

\section{Materials.}

All solvents and reagents were analytical-grade and used as received unless noted otherwise. Dimethyl sulfoxide (DMSO), concentrated hydrochloric acid $(\mathrm{HCl})$, cetyltrimethylammonium bromide (CTAB), triethylamine (TEA), sodium nitrite, diethylenetriaminepentaacetic acid (DPTA), and diisopropylethylamine (DIPEA) were purchased from Sigma Aldrich (St. Louis, MO). Anhydrous $N, N$-dimethylformamide (DMF), anhydrous tetrahydrofuran (THF), ammonium hydroxide $\left(\mathrm{NH}_{4} \mathrm{OH}, 28 \mathrm{wt} \%\right)$, methanol, $(\mathrm{MeOH})$ and ethanol $(\mathrm{EtOH})$ were purchased from Fisher Scientific (Fair Lawn, NJ). 5,5'-dithiobis-(2-nitrobenzoic acid) (Ellman's reagent) was purchased from Invitrogen Molecular Probes (Eugene, OR). Tetraethylorthosilicate (TEOS) and 3mercaptopropyltrimethoxysilane (MPTMS) were purchased from Gelest (Morrisville, PA) and stored under nitrogen atmosphere. Polyurethanes HP-93A and PC35-85A were received from Lubrizol (Cleveland, OH). Polyurethane AL25-80A was received from AdvanSource Biomaterials (Wilmington, MA). Nitrogen $\left(\mathrm{N}_{2}\right)$, argon (Ar), and nitric oxide calibration gas (NO, $25.87 \mathrm{ppm}$ in $\mathrm{N}_{2}$ ) were purchased from Airgas National Welders (Raleigh, NC). Water was purified using a Millipore Reference water purification system (Bedford, MA) to a resistivity of $18.2 \mathrm{M} \Omega \mathrm{cm}$ and a total organic content of $<6 \mathrm{ppb}$.

\section{Synthesis of NO-releasing silica nanoparticles.}

Synthesis of thiol-functionalized nonporous silica nanoparticles was carried out through the Stöber method using MPTMS and TEOS, as previously described. ${ }^{26}$ Briefly, a MPTMS:TEOS mixture was added dropwise to a solution of ethanol, water and ammonia. The molar ratio of reactants (EtOH: $\mathrm{H}_{2} \mathrm{O}: \mathrm{NH}_{4} \mathrm{OH}: M P T M S: T E O S$ ) was 333:91.8:561:3:1. The reaction was allowed to proceed for $2 \mathrm{~h}$, followed by washing of the particles with ethanol, and particle collection by centrifuging three times. 
The synthesis of mesoporous silica nanoparticles (MSN) was achieved by combining 175 $\mathrm{mL}$ EtOH, $162 \mathrm{~mL} \mathrm{H}_{2} \mathrm{O}, 11.8 \mathrm{~mL} \mathrm{NH}_{4} \mathrm{OH}$, and $280 \mathrm{mg} \mathrm{CTAB} .{ }^{35}$ This solution was stirred for $\sim 15 \mathrm{~min}$ to form the liquid crystal template necessary for the generation of porous particles. The TEOS $(1.395 \mathrm{~mL})$ was added as a bolus with additional stirring $(2 \mathrm{~h})$ at room temperature. The solution became cloudy white, indicating particle formation. Particles were washed three times with ethanol and collected by centrifugation. Following formation of the TEOS MSNs, CTAB still present in the pores was removed via an ion exchange with ethanolic hydrochloric acid. Particles were dried under vacuum, then treated with oxygen plasma for $2 \mathrm{~h}$ to expose surface silanols, facilitating maximum functionalization in subsequent steps. Particles at this point in the synthesis are referred to as pre-grafted MSNs.

To functionalize the MSNs with thiol groups, a mercaptosilane (MPTMS) was surface grafted onto the bare TEOS MSN scaffold. Approximately $50 \mathrm{mg}$ of MSNs were suspended in $20 \mathrm{~mL}$ anhydrous DMF, followed by the addition of $1 \mu \mathrm{L}$ TEA/mg MSN. The TEA served as a base catalyst. The solution was sonicated to distribute the particles before adding 130 $\mu \mathrm{L}$ MPTMS/mg MSN as a bolus. The flask was immediately set to reflux at $150{ }^{\circ} \mathrm{C}$ for $12 \mathrm{~h}$. The solution was then cooled to room temperature, particles were collected by centrifugation with ethanol wash three times, and dried under vacuum. The particles at this stage are referred to as post-grafted MSNs.

The next step involved nitrosating the particles via exposure to acidified nitrite. Thiolmodified particles $(25 \mathrm{mg}$ ) were dissolved in a mixture of $5 \mathrm{~mL} \mathrm{MeOH}$ and $1 \mathrm{~mL} \mathrm{HCl} \mathrm{(5}$ M). An aqueous solution $(500 \mu \mathrm{L})$ of nitrite $(50 \mathrm{mg})$ and DPTA $(10 \mathrm{mg})$ was then added slowly to the particle solution. This mixture was shielded from light and stirred at $0{ }^{\circ} \mathrm{C}$ for 1 h. Particles were then collected by centrifugation and washed with $-20{ }^{\circ} \mathrm{C} \mathrm{MeOH}$ three times. The particle batches were dried under vacuum for $45 \mathrm{~min}$ to remove all remaining $\mathrm{MeOH}$. Nitrosated particles, deep pink in color, were used immediately after removal from the vacuum box. Care was exercised to minimize ambient light exposure during the nitrosation process, as light may prompt the premature release of NO.

\section{Silica nanoparticle physiochemical characterization.}

Particle morphology and geometric size were determined using a Hitachi S-4700 Cold Cathode Field Emission Scanning Electron Microscope (Pleasanton, CA). Samples in scanning electron micrographs were sputter-coated with $5.0 \mathrm{~nm}$ gold/platinum. Specific surface area and pore width of the MSNs were obtained via nitrogen sorption isotherms using a Micrometrics Tristar II 3020 Surface Area and Porosity Analyzer (Norcross, GA). Porosimeter samples were dried at $115^{\circ} \mathrm{C}$ for $12 \mathrm{~h}$ prior to analysis. The specific surface area was assessed using the Brunauer-Emmett-Teller (BET) method using the adsorption isotherm over the $\mathrm{p} / \mathrm{p}^{\circ}$ range of $0.05-0.15$. The pore width was measured over the $\mathrm{p} / \mathrm{p}^{\circ}$ range of 0.05-0.60 using the Barrett-Joyner-Halenda (BJH) method. Zeta potential of the particles was determined using a Zetasizer Nano ZS Particle Size and Zeta Potential Dynamic Light Scattering Instrument (Malvern, U.K.). Samples were suspended at a concentration of $1 \mathrm{mg}$ $\mathrm{mL}^{-1}$ in phosphate buffer at $\mathrm{pH} 7.4$ and sonicated directly prior to analysis. The Ellman's assay was used to determine the free thiol content of modified particles. ${ }^{26,36}$ Known masses of particles were added to a solution of DMSO $(2.5 \mathrm{~mL}), \mathrm{MeOH}(2 \mathrm{~mL})$, DIPEA $(20 \mu \mathrm{L})$, 
and $10 \mathrm{mM}$ Ellman's reagent (1.5 mL, in DMSO). A calibration curve was constructed using known concentrations of L-cysteine. Samples were incubated at room temperature for $1 \mathrm{~h}$, followed by absorbance measurements at $412 \mathrm{~nm}$ using a Labsystems MultiskanRC plate reader (Helsinki, Finland).

\section{Suspension of porous and nonporous particles in polyurethane membranes.}

Polyurethane solutions were prepared by dissolving polyurethane at a concentration of 80 $\mathrm{mg} \mathrm{mL}^{-1}$ into 3:1 anhydrous THF:DMF, and sonicating at $60{ }^{\circ} \mathrm{C}$. Upon full dissolution of the polymer, the solution was cooled to room temperature and particles dispersed into the solution at a range of concentrations ( $10 \mathrm{mg} \mathrm{mL}^{-1}$ to $80 \mathrm{mg} \mathrm{mL}^{-1}$ ), sonicating vigorously to insure homogenous dispersal. Polyurethane membranes were deposited by a loop-casting method in the dark on a stainless steel wire to provide uniform coatings of polyurethane. For each layer, $6.5 \mu \mathrm{L}$ of the polyurethane solution was pipetted onto a $2 \mathrm{~mm}$ steel wire loop. This loop was passed over the wire for a total of seven separate coats, with a drying time of 5 min between each coat. An identical coating technique was used to coat sealed glass capillaries to assess the impact of trace metal ions on NO-release rates. After all coats had been applied, membranes were dried for an additional $1 \mathrm{~h}$, and then stored at $-20{ }^{\circ} \mathrm{C}$ until use.

\section{Nitric oxide-release measurements.}

Nitric-oxide release was measured via two methods. Instantaneous NO-release was measured in real-time using a Sievers 280i Chemiluminescent Nitric Oxide Analyzer (NOA; $\mathrm{CO})$. The NOA was calibrated before use with a two-point calibration consisting of air passed through an $\mathrm{NO}$ zero filter and $25.87 \mathrm{ppm} \mathrm{NO}$ in $\mathrm{N}_{2}$ as blank and standard values, respectively. When measuring NO release, either $1 \mathrm{mg}$ of particles or one $7-\mathrm{cm}$ polyurethane-coated wire was added to a sample flask with $30 \mathrm{~mL}$ deoxygenated $\mathrm{PBS}$ at $\mathrm{pH}$ 7.4 and $37^{\circ} \mathrm{C}$. The sample flask was shielded from light using aluminum foil to restrict undesirable light-initiated NO release. Nitrogen gas was bubbled through the sample solution at $80 \mathrm{~mL} \mathrm{~min}{ }^{-1}$ to transport liberated NO from the solution to the instrument reaction cell. Measurements were taken until the instrument value fell below a limit of detection of $6 \mathrm{ppb} \mathrm{mg} \mathrm{particles}{ }^{-1} \mathrm{~s}^{-1}$ for particles or $0.8 \mathrm{pmol} \mathrm{cm} \mathrm{membrane} \mathrm{c}^{-2} \mathrm{~s}^{-1}$ for particle-doped polyurethanes.

Due to the low, extended NO flux of the RSNO-modified films, an NO-measurement technique with a lower limit of detection was required to better characterize the NO flux profile. The Griess assay was thus used to quantify low-flux NO release as previously described. ${ }^{37}$ Briefly, polyurethane-coated wires were incubated in PBS at $\mathrm{pH} 7.4$ at $37{ }^{\circ} \mathrm{C}$ in the dark, with aliquots of the soak solution removed at various time-points over the samples' release duration. These samples were assessed by mixing $50 \mu \mathrm{L}$ sample solution, $50 \mu \mathrm{L}$ $0.1 \% \mathrm{w} / \mathrm{v}$ aqueous $N$-(1-napthyl)ethylene diamine, and $50 \mu \mathrm{L} 1 \% \mathrm{w} / \mathrm{v}$ sulfanilamide in $5 \%$ $\mathrm{v} / \mathrm{v}$ aqueous phosphoric acid. This mixture was incubated for $5 \mathrm{~min}$ to allow formation of the colored azo dye. Absorbance of the sample solutions was measured at $540 \mathrm{~nm}$ on a Labsystem MultiskanRC Microplate Spectrophotometer (Helsinki, Finland). A calibration curve of nitrite from $2-100 \mu \mathrm{M}$ allowed for indirect quantitative determination of the NO 
concentration in the polyurethane soak solutions. For the thermal stability study, polyurethane films were stored in microcentrifuge tubes sealed in opaque bags.

\section{Film leaching analysis.}

Leaching of the RSNO-modified particles from polyurethane films was characterized using a Teledyne-Leeman Laboratories Prodigy High Dispersion inductively coupled plasma optical emission spectroscopy (ICP-OES; Hudson, NH). Wires coated with particle-doped polyurethane membranes were incubated in PBS at $\mathrm{pH} 7.4$ at $37{ }^{\circ} \mathrm{C}$ in the dark. After $21 \mathrm{~d}$, the polyurethane-coated wires were removed and the soak solution was analyzed for silicon content, using the Si emission line at $251.611 \mathrm{~nm}$. Calibration was performed using both sodium silicate standards and MSN standards, with a linear response confirmed from both standard sets over a range of 0.1 to $25 \mathrm{ppm}$ in PBS. The buffer solution used in this experiment was prepared using only polypropylene vessels, as silicic acid from glass containers was previously shown to reach the soak solution and display measurable Si levels. 38

\section{Results and Discussion}

\section{S-nitrosothiol-functionalized mesoporous silica nanoparticles.}

The synthesis of $S$-nitrosothiol-functionalized silica nanoparticles with nonporous, hollow, diatomaceous, and fumed morphologies have previously been reported by multiple synthetic techniques. ${ }^{26,27,39,40}$ In addition, confined microenvironments provided by organic polymers have been shown to lead to greater RSNO radical pair recombination compared to those with less rigid milieus. ${ }^{41}$ We thus hypothesized that a novel and effective path for enhancing the stability of RSNO-functionalized nanoparticles may result from porosity within a silica particle network. Likewise, the increased specific surface area of MSNs might allow for larger NO payloads as compared to nonporous analogues.

To take advantage of the impact of mesoporosity on NO stabilization, a protocol was developed to functionalize silica nanoparticles with RSNO donors both on the exterior surface and within the pores. In this manner, the mercaptosilanes were post-grafted onto the MSNs to achieve greater functional group grafting density. ${ }^{42}$ Briefly, mesoporous silica nanoparticles made entirely of the backbone silane TEOS were synthesized through a modified Stöber method in the presence of a liquid crystal surfactant template. ${ }^{35}$ After particle formation, the surfactant was removed by ion exchange with acid, allowing the surface silanols to be activated by oxygen plasma treatment. Finally, the MSNs were postgrafted with a primary mercaptosilane, MPTMS, by refluxing in base-catalyzed DMF. Optimization of the synthetic procedure identified ideal reflux solvent, reflux temperature, and mercaptosilane concentration in order to achieve monodisperse, densely-grafted RSNOmodified MSNs.

The MSNs were characterized before and after MPTMS grafting (Table 1). Zeta potential measurements revealed that the surface of the particles was more neutral after modification, demonstrating the conversion of anionic surface silanols to neutral thiol end-groups. Of note, zeta potential measurements only provide evidence of exterior particle surface modification. 
Porosimetry measurements indicated that the surface area of the particle was decreased by almost half after grafting (1041 to $549 \mathrm{~m}^{2} \mathrm{~cm}^{-1}$ ), validating intraporous grafting. ${ }^{35,43}$ Grafting on both the exterior and interior particle surface was confirmed through a combination of surface charge and surface area characterization methods. Grafting inside of the pores is essential for taking advantage of both the large surface area characteristic of the MSNs and the stability provided by the confined intraporous structure.

\section{Morphology and NO-release comparison of porous and nonporous particles.}

The impact of particle pores on the NO release was elucidated for the first time by comparing the NO-releasing kinetics of RSNO-functionalized nonporous and porous silica nanoparticles. To isolate the effect of the porous structure from that of other morphological characteristics, synthetic parameters were optimized so that the shape and size of both the nonporous and porous particles were identical. Morphological data on the particle size, surface charge, and specific surface area is provided in Table 2. Scanning electron micrographs of the nonporous particles and porous particles pre- and post-grafting allowed visual comparison and confirmation that each particle type had spherical, monodisperse morphologies (Figure 1). High monodispersity was also indicated by light scattering measurements $(\sim 1 \mu \mathrm{m}$ average size; $\mathrm{PDI}<0.1)$. In terms of surface characteristics, the zeta potential was the major difference between the two particles. While the zeta potential of the nonporous system was quite negative $(-30.9 \mathrm{mV})$, the porous particles were more neutral $(-5.4 \mathrm{mV})$. The negative surface charge observed for both particle systems is attributed to anionic surface silanols. The more neutral zeta potential of porous particles indicates greater conversion of negative silanols to neutral thiol end-groups during mercaptosilane functionalization. This difference in the degree of functionalization was confirmed via Ellman's assay, a method that quantifies free thiols. Indeed, the thiol content of the porous particles was more than double that of nonporous particles. From a morphological standpoint, the two particle systems have similar size, shape, and monodispersity but varying surface charge due resulting from the degree of surface modification.

The NO-release kinetics of the two particle systems were compared to evaluate the impact of pore structure on NO storage and stability. Nitric oxide-release parameters were determined using two complementary methods. While chemiluminescent detection using a Nitric Oxide Analyzer (NOA) allows for real-time NO flux measurements, dynamic initial flux profile, and release kinetics (e.g., half-life) with a relatively low limit of detection (nM), this instantaneous analysis method is not well suited for measuring the lower magnitude NO release typical of stabilized RSNO donors at extended periods. For this reason, the Griess assay, an indirect colorimetric assay that quantifies NO concentrations, was also used to assess NO-release over extended periods. Though the Griess assay has a higher limit of detection $(0.5 \mu \mathrm{M})$, it is only limited by the ability to differentiate between cumulative $\mathrm{NO}$ concentrations at successive time points, which allowed profiling of lower NO fluxes. Together, the full NO-release profiles of the NO-releasing systems were determined, including total payload $\left([\mathrm{NO}]_{\mathrm{T}}\right)$, maximum flux $\left([\mathrm{NO}]_{\max }\right)$, half-life $\left(\mathrm{t}_{1 / 2}\right)$, and release duration $\left(\mathrm{t}_{\mathrm{d}}\right) \cdot{ }^{44}$ 
As shown in Table 3, the NO release from the two RSNO-functionalized silica nanoparticles systems was markedly different. Overall, the porous particles had a larger NO payload, longer half-life, and greater release duration than the nonporous analogues. The free thiol content of porous particles correlates directly with the NO payload as more surface thiol groups are available for RSNO conversion. Even after incubation in PBS at $37{ }^{\circ} \mathrm{C}$ for $\sim 100$ $\mathrm{h}$, the particles still appeared slightly pink to the eye, evidence of intact primary RSNO groups. These results suggest that intraporous RSNO groups may be stabilized to the extent that their decomposition to $\mathrm{NO}$ is inhibited even under incubation conditions that should facilitate RSNO breakdown. This hypothesis was tested by exposing the particles to a $10 \mu \mathrm{M}$ $\mathrm{CuBr}_{2}$ solution; the available $\mathrm{Cu}^{2+}$ initiates catalytic decomposition of the RSNOs. ${ }^{27,45}$ Copper-based decomposition of RSNOs would neither occur via a radical process nor be influenced by a cage effect. A total purge of NO would be anticipated upon RSNO breakdown. As expected, the observed NO payload from the porous particles upon exposure to copper ions increased by $>50 \%$ to $3.29 \mu \mathrm{mol} \mathrm{mg}{ }^{-1}$, while the observed NO payload of the nonporous particles stayed largely the same. The results of the copper-initiated release support the hypothesis that the porous particles do not fully release all of their NO payload under the original incubation conditions. Even though some NO was retained by the MSN scaffold, the NO payload for the porous particles was still nearly twice that of their nonporous counterparts, a clear advantage of porous particles for drug delivery applications.

Another major difference between the two particle systems is their NO-release kinetics. The porous particles were characterized as having a 26-h half-life and total release duration of nearly four days, representing an unprecedented length of NO release. Generally, the mobility of RSNO groups largely dictates NO-release rates, as a thiyl radical must react with an intact RSNO group to avoid recombination with the NO radical. ${ }^{46}$ In this case, the significant difference in NO release (rates) between porous and nonporous particles of the same size and shape suggests that the porous structure also contributes to the prolonged release durations. Primary RSNO groups within the interior pore surface may exist in solvent cages enforced by the surrounding pore structure. When a confined, intraporous RSNO group undergoes photothermal degradation, the likelihood of recombination of the thiyl and NO radicals before NO can diffuse away appears greater than for exterior RSNO groups. The more extended NO-release profile from porous particles also corresponded to a lower maximum NO flux relative to the nonporous particles. Large NO fluxes, as displayed by the nonporous particles and nearly all $N$-diazeniumdiolate-based silica nanoparticles, generally limit NO-release duration. Additionally, large NO fluxes are associated with proinflammatory and cytotoxic processes including apoptotic responses and full cell cycle arrest. ${ }^{47-49}$ Thus, the low magnitude, extended NO flux achieved using porous particles holds promise for medical device applications where concerns over lowering inflammation, mitigating infections, and stimulating blood vessel growth are of importance.

\section{Polyurethane-based NO release using porous and nonporous particles.}

Dispersion of the silica nanoparticles in a polyurethane matrix was characterized to assess the ability to create stable NO-release polymers for medical devices. Polyurethane (PU) is a common biomedical material resulting from its inherent bioinertness and robust mechanical properties. ${ }^{50,51}$ Furthermore, polyurethane is available in a range of hydrophobicities to 
control water uptake. For example, hydrophobic PU coatings have been shown to reduce the rate of biofouling, thrombosis, and bacterial colonization on catheters, stents, and insulin pumps. ${ }^{52-54}$ Conversely, hydrophilic polyurethanes are important for certain biosensor designs, where the diffusion of analytes through the polymer matrix is essential for device functionality. ${ }^{55,56}$ An NO-releasing system capable of consistent NO release across a range of hydrophobicities would potentially allow for expanded medical device utility. Three polyurethane formulations were used as models of very hydrophilic (HP-93A), moderately hydrophilic (AL25-80A), and very hydrophobic (PC35-85A) PUs. Nitrosated nonporous and porous particles dispersed in these polyurethane solutions were loop cast onto wire substrates. Loop casting results in a reproducible thin, uniform polymeric layer containing particles. ${ }^{23,57}$

Nitric oxide release from membranes cast on analogous glass substrates were consistent with those on the steel wire substrates, indicating that any trace metal ions from the wires did not impact NO release (Table S1). Table 4 depicts the NO-release characteristics as a function of polyurethane type.

Particles doped into polyurethanes were characterized as having prolonged NO-release durations and decreased NO fluxes relative to native particles. These changes are attributed to the polymer matrix serving to confine both exterior and intraporous RSNO groups. Little difference between the NO-release kinetics of the three different particle-doped membranes was observed, even though the three polyurethanes tested essentially span the range of possible hydrophobicities. While the maximum NO flux did not trend with hydrophobicity, the three polyurethanes displayed $[\mathrm{NO}]_{\max }$ values almost an order of magnitude lower than the native particles (Table 3). The confinement imposed by the polyurethane matrix may suppress rapid degradation of exterior RSNO groups responsible for the initial flux of NO from native particles. The relatively limited NO-release durations from polyurethanes doped with nonporous particles emphasizes the benefit of a pore-associated stabilization to extend release durations in particle-doped polyurethanes (Table S2). The duration of NO release also appeared to be independent of polyurethane water uptake properties as the NO half-life and release duration did not trend with polyurethane hydrophobicity. Therefore, RSNObased NO release may be especially attractive for medical device coatings. Indeed, a polyurethane composition may be selected based on the particular design criteria of a given device application, not by an NO-release mechanism.

\section{Temperature stability and leaching of MSN-doped polyurethanes.}

Medical device coatings necessitate robust materials, both prior to and after implantation in the body. The thermal lability of RSNO-particle-doped PU was thus characterized under different storage conditions. Often, the instability of RSNO-based NO-release systems under ambient temperature conditions is pointed to as a shortcoming or disadvantage compared to $\mathrm{N}$-diazeniumdiolate NO donors. Membranes capable of ambient temperature storage would make the use of RSNO-based device coatings more appealing. Thermal stability testing was carried out by exposing light-shielded RSNO-modified MSN-doped HP-93A polyurethane membranes to a range of storage conditions $\left(-20^{\circ} \mathrm{C}, 0{ }^{\circ} \mathrm{C}\right.$ and $\left.23{ }^{\circ} \mathrm{C}\right)$ for $96 \mathrm{~h}$. Prior work has shown that cold storage results in better retention of NO payloads. ${ }^{28,32}$ 
As expected, room temperature storage resulted in reduced NO payloads (Figure 2), while membranes stored at -20 and $0{ }^{\circ} \mathrm{C}$ showed negligible NO payload loss out to $96 \mathrm{~h}$. While storage at room temperature eventually leads to lower NO payloads, storage at $0{ }^{\circ} \mathrm{C}$ is sufficient for maintaining NO payloads for at least $4 \mathrm{~d}$. Nonporous particle-doped polyurethanes had similar relative thermal stability versus porous particle-doped polyurethanes (Figure S1). The formation of a solvent cage requires the presence of solvent, so the cage effect would not significantly contribute to RSNO stabilization during dry storage. Instead, the increased thermal stability of these NO-releasing materials at lower temperatures is more likely due to decreased thermal mobility of the RSNO functional groups. Overall, enhanced thermal stability is essential for practical NO-release applications, such as those involving medical device coatings.

The stability of the RSNO-modified MSN-doped membranes was also assessed with respect to the final fate of the nanoparticles (i.e., leaching). While silica nanoparticles are often used for their bioinertness, silica can still provoke oxidative stress, tissue injury, and endothelial dysfunction. ${ }^{58-60}$ The best strategy for avoiding undesirable nanoparticle-associated toxicity is to minimize particle leaching from the membranes.

$S$-nitrosothiol-modified MSN-doped polyurethane membranes were incubated at $37{ }^{\circ} \mathrm{C}$ in PBS at $\mathrm{pH} 7.4$ for $21 \mathrm{~d}$. The extent of particle leaching was determined via elemental analysis (ICP-OES) of silicon in the leachate solution (Figure 3). Comparing across the types of polyurethane, with a constant MSN concentration, the greatest degree of leaching was observed from the most hydrophilic polyurethane, HP-93A. Hydrophilic polyurethanes are known to swell upon water uptake, increasing particle displacement (i.e., leaching). ${ }^{61} \mathrm{To}$ understand the dependence of particle concentration in the membrane on leaching, HP-93A polymers containing 10 to $80 \mathrm{mg} \mathrm{mL}^{-1}$ were soaked in buffer for $21 \mathrm{~d}$. A concentration of $80 \mathrm{mg} \mathrm{mL}^{-1}$ particles in the HP-93A was the largest dopant amount tested as polyurethane did not form a continuous membrane around particles at higher concentrations. While $80 \mathrm{mg}$ $\mathrm{mL}^{-1}$ membranes demonstrated the greatest percentage of particles leached, leaching from the membranes was at least partially independent of particle concentration. As shown in Figure 3, the least leaching was observed for $20 \mathrm{mg} \mathrm{mL}^{-1}$ particles, while 10 and $40 \mathrm{mg} \mathrm{mL}$ ${ }^{-1}$ particles were statistically equivalent. Increasing hydrophilicity and particle concentration both corresponded with marginally greater leaching magnitudes.

Each of the particle-doped polyurethane compositions tested showed negligible leaching (i.e., $<1 \mathrm{wt} \%$ leached particles after $21 \mathrm{~d}$ incubation). The percentage of particles leached corresponds to a total mass of less than $10 \mu \mathrm{g} \mathrm{cm}{ }^{-2}$, a level unlikely to provoke any significant local tissue toxicity. ${ }^{60}$ The degree of SNP leaching has often been attributed to both total particle electrical charge and particle surface hydrophobicity, with previous reports suggesting that a high degree of alkanethiol modification counteracts leaching common to silica nanoparticles. ${ }^{38,61}$ As the degree of thiol modification of the MSNs described is great, the net particle surface charge is almost neutral (Table 1). Furthermore, the particles are hydrophobic due to the nature of the alkanethiol functional groups decorating their surface. This surface hydrophobicity is distinct from that of more highly charged particles (e.g., amine-based NONOates) known to leach over shorter soak periods. ${ }^{38}$ This negligible leaching may allow for expanded use of RSNO-doped polymeric membranes 
over extended periods, which has not been possible with alternative $N$-diazeniumdiolate NO donors.

\section{CONCLUSION}

The synthesis of monodisperse, densely-grafted RSNO-functionalized MSNs capable of continuously releasing NO for $90 \mathrm{~h}$ represents unprecedented release duration from a nanoparticle scaffold. The remarkable stability demonstrated by RSNO-functionalized MSNs is attributed to an enhanced confinement of intraporous NO donor moieties. As this stabilization effect is functionally independent of water uptake into the membrane, RSNOfunctionalized MSNs enable extended NO-release profiles from materials regardless of their water uptake or wettability. Polymeric NO-release durations of $>30 \mathrm{~d}$ are achievable, without particle leaching or the need for extremely cold storage conditions. These attributes present new opportunities for introducing the benefits of $\mathrm{NO}$ to wider range of medical devices, where polyurethane composition selection is often tied to a given device's need or utility.

\section{Supplementary Material}

Refer to Web version on PubMed Central for supplementary material.

\section{ACKNOWLEDGEMENTS}

This work was performed in part at the Chapel Hill Analytical and Nanofabrication Laboratory, CHANL, a member of the North Carolina Research Triangle Nanotechnology Network, RTNN, which is supported by the National Science Foundation, Grant ECCS-1542015, as part of the National Nanotechnology Coordinated Infrastructure, NNCI.

Funding Sources

This research was supported by the National Institutes of Health (DK108318)

\section{REFERENCES}

(1). Witte MB; Barbul A Role of Nitric Oxide in Wound Repair. Am. J. Surg 2002, 183, 406-412. [PubMed: 11975928]

(2). Cooke JP NO and Angiogenesis. Atherosclerosis 2003, 4, 53-60. [PubMed: 14664903]

(3). Wang Y; Vaddiraju S; Gu B; Papadimitrakopoulos F; Burgess DJ Foreign Body Reaction to Implantable Biosensors. J. Diabetes Sci. Technol 2015, 9, 966-977. [PubMed: 26306495]

(4). Bogdan C Nitric Oxide and the Immune Response. Nat. Immunol 2001, 2, 907-916. [PubMed: 11577346]

(5). De Groote MA; Fang FC NO Inhibitions: Antimicrobial Properties of Nitric Oxide Oxford Univ. Press 1995, 21, 162-165.

(6). Hakim TS; Sugimori K; Camporesi EM; Anderson G Half-Life of Nitric Oxide in Aqueous Solutions with and without Haemoglobin. Physiol. Meas 1996, 17, 267-277. [PubMed: 8953625]

(7). Varu VN; Tsihlis ND; Kibbe MR Nitric Oxide-Releasing Prosthetic Materials. Vasc. Endovascular Surg 2009, 43, 121-131. [PubMed: 18799500]

(8). Seabra AB; Durán N Nitric Oxide-Releasing Vehicles for Biomedical Applications. J. Mater. Chem 2010, 20, 1624-1637.

(9). Carpenter AW; Schoenfisch MH Nitric Oxide Release: Part II. Therapeutic Applications. Chem. Soc. Rev 2012, 41, 3742-3752. [PubMed: 22362384] 
(10). Zanini S; Polissi A; Maccagni EA; Dell'Orto EC; Liberatore C; Riccardi C Development of Antibacterial Quaternary Ammonium Silane Coatings on Polyurethane Catheters. J. Colloid Interface Sci 2015, 451, 78-84. [PubMed: 25890115]

(11). Wrzeszcz A; Dittrich B; Haamann D; Aliuos P; Klee D; Nolte I; Lenarz T; Reuter G Dexamethasone Released from Cochlear Implant Coatings Combined with a Protein Repellent Hydrogel Layer Inhibits Fibroblast Proliferation. J. Biomed. Mater. Res. A 2014, 102, 442-454. [PubMed: 23533184]

(12). Norton LW; Koschwanez HE; Wisniewski NA; Klitzman B; Reichert WM Vascular Endothelial Growth Factor and Dexamethasone Release from Nonfouling Sensor Coatings Affect the Foreign Body Response. J. Biomed. Mater. Res. A 2007, 81, 858-869. [PubMed: 17236219]

(13). Koh A; Carpenter AW; Slomberg DL; Schoenfisch MH Nitric Oxide-Releasing Silica Nanoparticle-Doped Polyurethane Electrospun Fibers. ACS Appl. Mater. Interfaces 2013, 5, 7956-7964. [PubMed: 23915047]

(14). Chou HC; Chiu SJ; Liu YL; Hu TM Direct Formation of S-Nitroso Silica Nanoparticles from a Single Silica Source. Langmuir 2014, 30, 812-822. [PubMed: 24410024]

(15). Lu Y; Slomberg DL; Schoenfisch MH Nitric Oxide-Releasing Chitosan Oligosaccharides as Antibacterial Agents. Biomaterials 2015, 35, 1716-1724.

(16). Pegalajar-Jurado A; Wold KA; Joslin JM; Neufeld BH; Arabea KA; Suazo LA; McDaniel SL; Bowen RA; Reynolds MM Nitric Oxide-Releasing Polysaccharide Derivative Exhibits 8-Log Reduction against Escherichia Coli, Acinetobacter Baumannii and Staphylococcus Aureus. J. Control. Release 2015, 220, 617-623. [PubMed: 26686492]

(17). Sun B; Slomberg DL; Chudasama SL; Lu Y; Schoenfisch MH Nitric Oxide-Releasing Dendrimers as Antibacterial Agents. Biomacromolecules 2013, 13, 3343-3354.

(18). Rashti A; Yahyaei H; Firoozi S; Ramezani S; Rahiminejad A; Karimi R; Farzaneh K; Mohseni M; Ghanbari H Development of Novel Biocompatible Hybrid Nanocomposites Based on Polyurethane-Silica Prepared by Sol Gel Process. Mater. Sci. Eng. C 2016, 69, 1248-1255.

(19). Ibrahim IAM; Zikry AAF; Sharaf MA Preparation of Spherical Silica Nanoparticles: Stober Silica. J. Am. Sci 2010, 6, 985-989.

(20). Chen Y; Chen H; Shi J In Vivo Bio-Safety Evaluations and Diagnostic/Therapeutic Applications of Chemically Designed Mesoporous Silica Nanoparticles. Adv. Mater 2013, 25, 3144-3176. [PubMed: 23681931]

(21). Ren H; Colletta A; Koley D; Wu J; Xi C; Major TC; Bartlett RH; Meyerhoff ME Thromboresistant/Anti-Biofilm Catheters via Electrochemically Modulated Nitric Oxide Release. Anal. Chem 2015, 25, 368-379.

(22). Koh A; Nichols SP; Schoenfisch MH Glucose Sensor Membranes for Mitigating the Foreign Body Response. J. Diabetes Sci. Technol 2011, 5, 1052-1059. [PubMed: 22027297]

(23). Wolf AK; Qin Y; Major TC; Meyerhoff ME Improved Thromboresistance and Analytical Performance of Intravascular Amperometric Glucose Sensors Using Optimized Nitric Oxide Release Coatings. Chinese Chem. Lett 2015, 26, 464-468.

(24). Soto RJ; Privett BJ; Schoenfisch MH In Vivo Analytical Performance of Nitric Oxide-Releasing Glucose Biosensors. Anal. Chem 2014, 86, 7141-7149. [PubMed: 24984031]

(25). Slomberg DL; Lu Y; Broadnax AD; Hunter RA; Carpenter AW; Schoenfisch MH Role of Size and Shape on Biofilm Eradication for Nitric Oxide-Releasing Silica Nanoparticles. ACS Appl. Mater. Interfaces 2013, 5, 9322-9329. [PubMed: 24006838]

(26). Riccio DA; Nugent JL; Schoenfisch MH Stöber Synthesis of Nitric Oxide-Releasing SNitrosothiol-Modified Silica Particles. Chem. Mater 2011, 23, 1727-1735. [PubMed: 21499510]

(27). Frost MC; Meyerhoff ME Synthesis, Characterization, and Controlled Nitric Oxide Release from S-Nitrosothiol-Derivatized Fumed Silica Polymer Filler Particles. J. Biomed. Mater. Res 2005, 72, 409-419.

(28). De Oliveira MG; Shishido SM; Seabra AB; Morgon NH Thermal Stability of Primary SNitrosothiols: Roles of Autocatalysis and Structural Effects on the Rate of Nitric Oxide Release. J. Phys. Chem. A 2002, 106, 8963-8970.

(29). Rabinowitch E; Wood WC The Collison Mechanism and the Primary Photochemical Process in Solutions. Trans. Faraday Soc 1936, 32, 1381-1387. 
(30). Franck J; Rabinowitch E Free Radicals and the Photochemistry of Solutions. Trans. Faraday Soc 1934, 30, 120-130.

(31). Shishido SM; Seabra AB; Loh W; De Oliveira MG Thermal and Photochemical Nitric Oxide Release from S-Nitrosothiols Incorporated in Pluronic F127 Gel: Potential Uses for Local and Controlled Nitric Oxide Release. Biomaterials 2003, 24, 3543-3553. [PubMed: 12809783]

(32). Shishido SM; Oliveira MG Polyethylene Glycol Matrix Reduces the Rates of Photochemical and Thermal Release of Nitric Oxide from S-Nitroso-N-Acetylcysteine. Photochem. Photobiol 2007, 71, 273-280.

(33). Yang L; Lu Y; Soto RJ; Shah A; Ahonen MJR; Schoenfisch MH S-Nitrosothiol-Modified Hyperbranched Polyesters. Polym. Chem 2016, 7, 7161-7169.

(34). Stasko NA; Fisher TH; Schoenfisch MH S-Nitrosothiol-Modified Dendrimers as Nitric Oxide Delivery Vehicles. Biomacromolecules 2009, 6, 247-253.

(35). Soto RJ; Yang L; Schoenfisch MH Functionalized Mesoporous Silica via an Aminosilane Surfactant Ion Exchange Reaction: Controlled Scaffold Design and Nitric Oxide Release. ACS Appl. Mater. Interfaces 2016, 8, 2220-2231. [PubMed: 26717238]

(36). Ellman GL Tissue Sulfhydryl Groups. Arch. Biochem. Biophys 1959, 82, 70-77. [PubMed: 13650640]

(37). Koh A; Riccio DA; Sun B; Carpenter AW; Nichols SP; Schoenfisch MH Fabrication of Nitric Oxide-Releasing Polyurethane Glucose Sensor Membranes. Biosens. Bioelectron 2011, 28, $17-$ 24. [PubMed: 21795038]

(38). Soto RJ; Schofield JB; Walter SE; Malone-Povolny MJ; Schoenfisch MH Design Considerations for Silica-Particle-Doped Nitric-Oxide-Releasing Polyurethane Glucose Biosensor Membranes. ACS Sensors 2017, 2, 140-150. [PubMed: 28722434]

(39). Liu T; Zhang W; Yang X; Li C Hollow Polymer Nanoparticles with S-Nitrosothiols as Scaffolds for Nitric Oxide Release. J. Colloid Interface Sci 2015, 459, 115-122. [PubMed: 26275504]

(40). Grommersch BM; Pant J; Hopkins SP; Goudie MJ; Handa H Biotemplated Synthesis and Characterization of Mesoporous Nitric Oxide-Releasing Diatomaceous Earth Silica Particles. ACS Appl. Mater. Interfaces 2018, 10, 2291-2301. [PubMed: 29278482]

(41). Denisov ET Cage Effects in a Polymer Matrix. Macromol. Chem. Phys 1984, 8, 63-78.

(42). Minko S Grafting on Solid Surfaces: "Grafting to" and "Grafting from" Methods In Polymer Surfaces and Interfaces; 2008; pp 215-234.

(43). Kruk M; Jaroniec M Gas Adsorption Characterization of Ordered Organic-Inorganic Nanocomposite Materials. Chem. Mater 2001, 13, 3169-3183.

(44). Coneski PN; Schoenfisch MH Nitric Oxide Release: Part III. Measurement and Reporting. Chem. Soc. Rev 2012, 41, 3753-3758. [PubMed: 22362308]

(45). Stamler JS; Toone EJ The Decomposition of Thionitrites. Curr. Opin. Chem. Biol 2002, 6, 779785. [PubMed: 12470731]

(46). Williams DLH The Chemistry of S-Nitrosothiols. Acc. Chem. Res 1999, 32, 869-876.

(47). Thomas DD; Ridnour LA; Isenberg JS; Flores-Santana W; Switzer CH; Donzelli S; Hussain P; Vecoli C; Paolocci N; Ambs S; Colton CA; Harris CC; Roberts DD; Wink DA The Chemical Biology of Nitric Oxide: Implications in Cellular Signaling. Free Radic. Biol. Med 2008, 45, $18-$ 31. [PubMed: 18439435]

(48). Cleeter MW; Cooper JM; Darley-Usmar VM; Moncada S; Schapira AHV Reversible Inhibition of Cytochrome c Oxidase, the Terminal Enzyme of the Mitochondrial Respiratory Chain, by Nitric Oxide. Implications for Neurodegenerative Diseases. Fed. Eur. Biochem. Soc. Lett 1994, 345, 50-54.

(49). Frungillo L; De Oliveira JFP; Saviani EE; Oliveira HC; Martínez MC; Salgado I Modulation of Mitochondrial Activity by S-Nitrosoglutathione Reductase in Arabidopsis Thaliana Transgenic Cell Lines. Biochim. Biophys. Acta 2013, 1827, 239-247. [PubMed: 23201478]

(50). Akindoyo JO; Beg MDH; Ghazali S; Islam MR; Jeyaratnam N; Yuvaraj AR Polyurethane Types, Synthesis and Applications-a Review. RSC Adv 2016, 6, 114453-114482.

(51). Davis FJ; Mitchell GR Polyurethane Based Materials with Applications in Medical Devices In Bio-Materials and Prototyping Applications in Medicine; 2008; pp 27-48. 
(52). Seo E; Na K Polyurethane Membrane with Porous Surface for Controlled Drug Release in Drug Eluting Stent. Biomater. Res 2014, 18, 15. [PubMed: 26331066]

(53). Treter J; Macedo AJ Catheters: A Suitable Surface for Biofilm Formation In Science Against Microbial Pathogens: Communicating Current Research and Technological Advances; 2011; pp 835-842.

(54). Haj-Ahmad R; Khan H; Arshad MS; Rasekh M; Hussain A; Walsh S; Li X; Chang MW; Ahmad Z Microneedle Coating Techniques for Transdermal Drug Delivery. Pharmaceutics 2015, 7, 486502. [PubMed: 26556364]

(55). Vadgama P; Peteu S Detection Challenges in Clinical Diagnostics; RSC Publishing, 2013.

(56). Fischer U Fundamentals of Glucose Sensors. Diabet. Med 1991, 8, 309-321. [PubMed: 1830254]

(57). Ward WK; Jansen LB; Anderson E; Reach G; Klein JC; Wilson GS A New Amperometric Glucose Microsensor: In Vitro and Short-Term in Vivo Evaluation. Biosens. Bioelectron 2002, 17, 181-189. [PubMed: 11839471]

(58). Murugadoss S; Lison D; Godderis L; Van Den Brule S; Mast J; Brassinne F; Sebaihi N; Hoet PH Toxicology of Silica Nanoparticles: An Update. Arch. Toxicol 2017, 91, 2967-3010. [PubMed: 28573455]

(59). Yu T; Greish K; McGill LD; Ray A; Ghandehari H Influence of Geometry, Porosity, and Surface Characteristics of Silica Nanoparticles on Acute Toxicity: Their Vasculature Effect and Tolerance Threshold. ACS Nano 2012, 6, 2289-2301. [PubMed: 22364198]

(60). Lin Y-S; Haynes CL Impacts of Mesoporous Silica Nanoparticle Size, Pore Ordering,and Pore Integrity on Hemolytic Activity. J. Am. Chem. Soc 2010, 132, 4834-4842. [PubMed: 20230032]

(61). Dolatzadeh F; Jalili MM; Moradian S Influence of Various Loadings of Hydrophilic or Hydrophobic Silica Nanoparticles on Water Uptake and Porosity of a Polyurethane Coating. Mater. Corros 2013, 64, 609-618. 

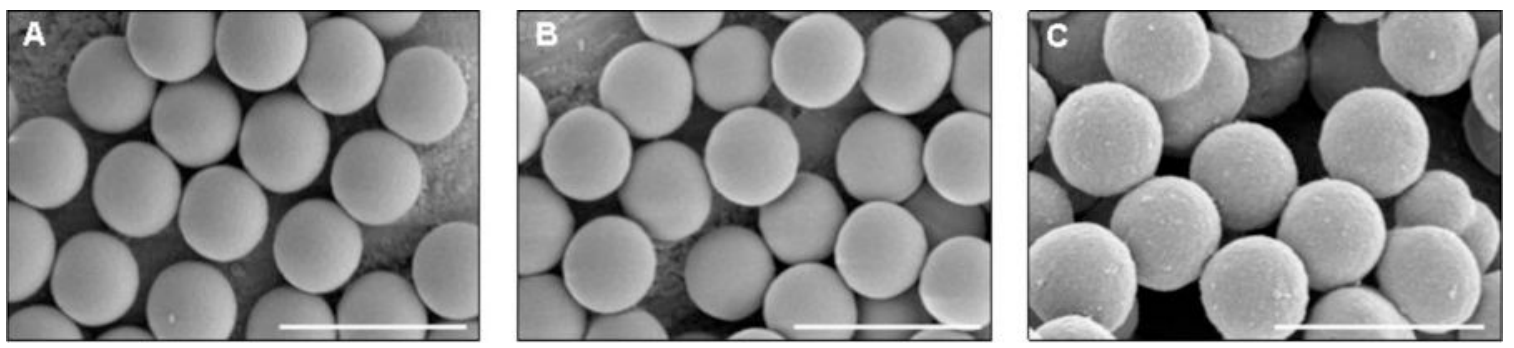

Figure 1.

Scanning electron micrographs of (A) bare TEOS MSNs; (B) MPTMS-functionalized MSNs; and (C) nonporous MPTMS:TEOS silica nanoparticles. Scale bars represent $2 \mu \mathrm{m}$. 


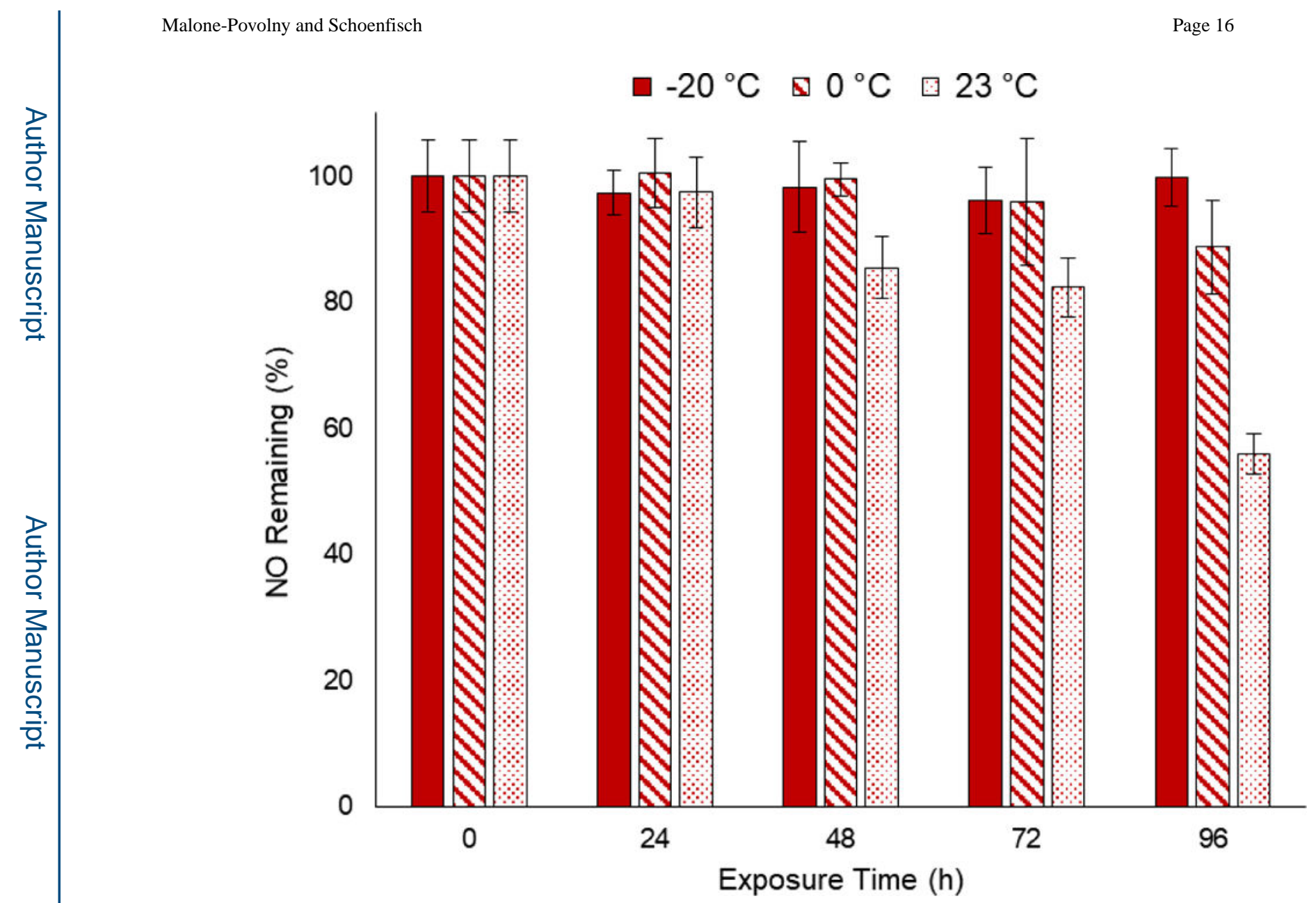

Figure 2.

Storage stability of RSNO-modified MSN-doped HP-93A polyurethane membranes over 96 $\mathrm{h}$ at -20 (solid), 0 (striped), and $23{ }^{\circ} \mathrm{C}$ (dotted). $n \succeq 6$ membranes. All significance is in reference to NO release prior to storage $(* \mathrm{p}<0.05 ; * * \mathrm{p}<0.01)$ 

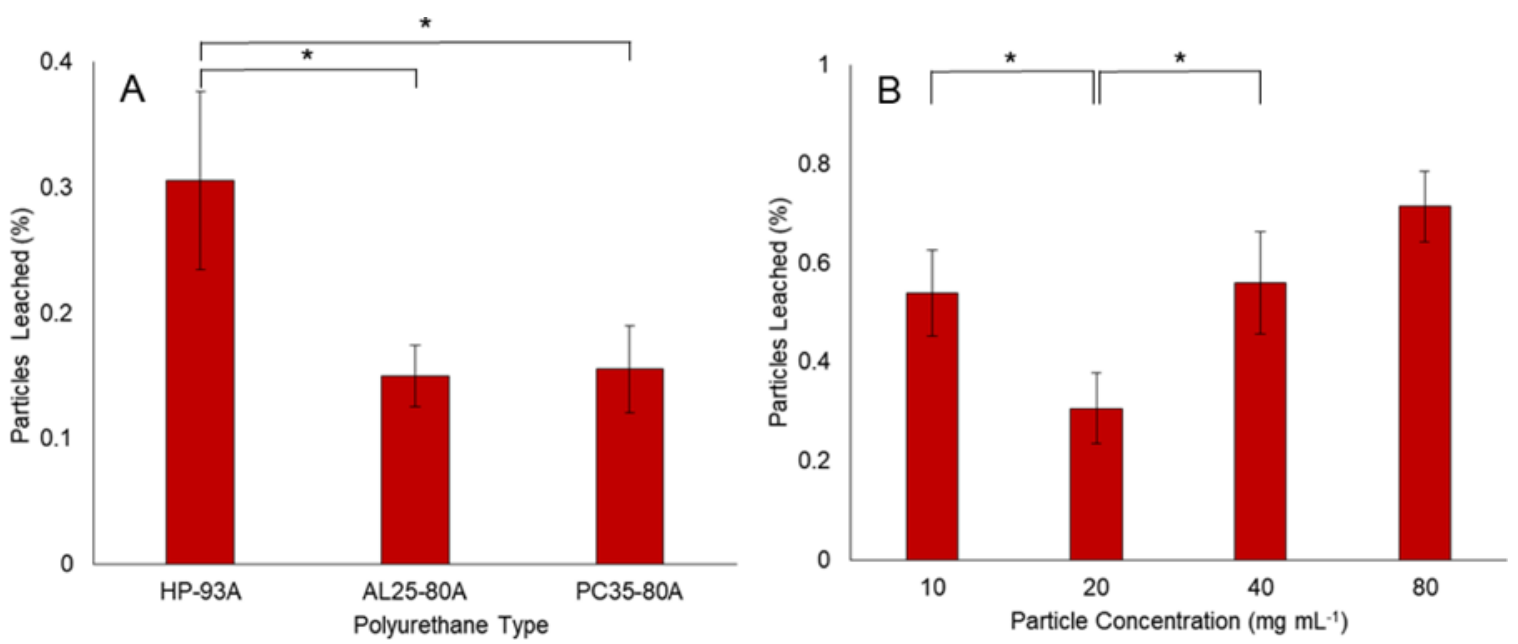

Figure 3.

Particle leaching as a function of (A) polyurethane membrane composition with $20 \mathrm{mg} \mathrm{mL}^{-1}$ MSN; and (B) MSN concentrations in HP-93A; $n \geq 6$ membranes after 21-d soaking in solution. $(* \mathrm{p}<0.05)$ 
Table 1.

Physiochemical characterization of pre- and post-grafted MPTMS:TEOS mesoporous silica nanoparticles. ${ }^{a}$

\begin{tabular}{cccc}
\hline MSN Synthesis Stage & Zeta Potential $(\mathbf{m V})$ & Pore Width $(\AA)$ & Specific Surface Area $\left(\mathbf{m}^{\mathbf{2}} \mathbf{g}^{\mathbf{- 1}}\right)^{\boldsymbol{c}}$ \\
\hline Pre-Grafting & $-32.8 \pm 1.7$ & $23.29 \pm 0.44$ & $1041 \pm 35$ \\
Post-Grafting & $-5.4 \pm 0.9$ & $20.77 \pm 1.63$ & $549 \pm 10$ \\
\hline
\end{tabular}

${ }^{a}$ Error bars represent standard deviation for $n \geq 3$ separate syntheses.

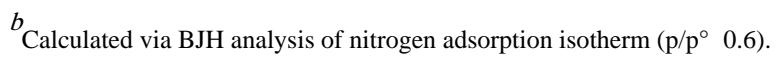

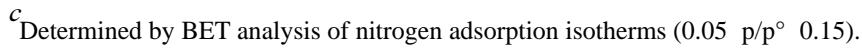


Table 2.

Comparison of nonporous and porous MPTMS:TEOS silica nanoparticle morphology. ${ }^{a}$

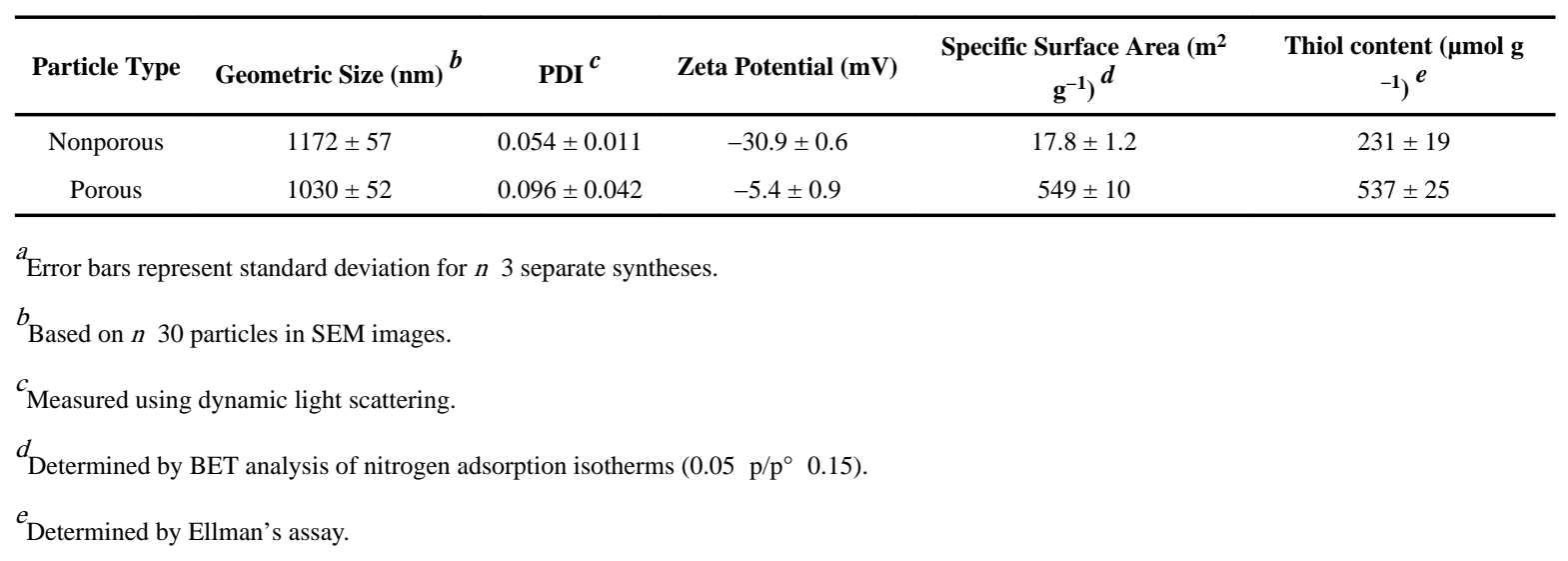


Table 3.

NO-release measurements in physiological buffer (PBS, pH 7.4, 37 ${ }^{\circ} \mathrm{C}$ ) of MPTMS:TEOS nanoparticles in the absence of light. ${ }^{a}$

\begin{tabular}{|c|c|c|c|c|c|}
\hline Particle Type & $\begin{array}{c}{[\mathrm{NO}]_{\max }} \\
\left(\mathrm{ppb} \mathrm{mg}^{-1}\right)^{b}\end{array}$ & $\mathbf{t}_{1 / 2}(\mathbf{h})^{c}$ & $\mathbf{t}_{\mathrm{d}}(\mathbf{h})^{d}$ & $\begin{array}{c}{[\mathrm{NO}]_{\mathrm{T}}} \\
\left(\mu \mathrm{mol} \mathrm{\textrm {mg } ^ { - 1 }}\right)^{e}\end{array}$ & $\begin{array}{c}{[\mathrm{NO}]_{\mathrm{Cu} 2+}} \\
\left(\mu \mathrm{mol} \mathrm{\textrm {mg } ^ { - 1 }} f\right.\end{array}$ \\
\hline Nonporous & $22800 \pm 2400$ & $2.92 \pm 0.91$ & $42.6 \pm 3.1$ & $1.55 \pm 0.17$ & $1.71 \pm 0.21$ \\
\hline Porous & $1040 \pm 80$ & $26.6 \pm 1.6$ & $89.1 \pm 2.5$ & $2.18 \pm 0.08$ & $3.29 \pm 0.14$ \\
\hline
\end{tabular}

E Error bars represent standard deviation for $n \geq 3$ separate syntheses.

${ }^{b}$ Maximum instantaneous NO flux.

$c_{\text {Half-life of NO release. }}$

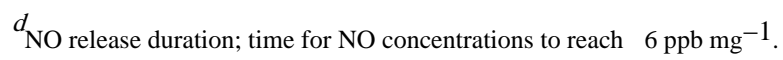

$e_{\text {Total NO release. }}$

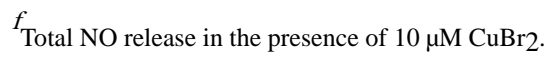


Table 4.

Nitric oxide-release measurements of polyurethane membranes doped with $80 \mathrm{mg} \mathrm{mL}^{-1}$ MPTMS:TEOS MSNs in physiological buffer (PBS, pH 7.4, $\left.37^{\circ} \mathrm{C}\right) .{ }^{a}$

\begin{tabular}{|c|c|c|c|c|c|}
\hline Polyurethane & $\begin{array}{c}\text { Water Uptake } \\
\left(\mathrm{mg} \mathrm{mg}^{-\mathbf{1}}\right)^{b}\end{array}$ & 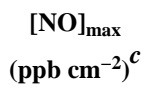 & $\mathrm{t}_{1 / 2}(\mathrm{~h})^{d}$ & $\mathrm{t}_{\mathrm{d}}(\mathrm{d}){ }^{e}$ & $\begin{array}{c}{[\mathrm{NO}]_{\mathrm{T}}} \\
\left(\mu \mathrm{mol} \mathrm{cm}^{-2}\right)^{f}\end{array}$ \\
\hline HP-93A & $2.61 \pm 0.12$ & $254 \pm 9$ & $37.8 \pm 3.1$ & $33.2 \pm 0.3$ & $4.18 \pm 0.24$ \\
\hline AL25-80A & $0.63 \pm 0.14$ & $286 \pm 14$ & $29.1 \pm 4.0$ & $30.7 \pm 0.7$ & $2.24 \pm 0.63$ \\
\hline PC $35-85 A$ & $0.18 \pm 0.06$ & $104 \pm 10$ & $24.0 \pm 2.2$ & $29.5 \pm 0.4$ & $3.94 \pm 0.39$ \\
\hline
\end{tabular}

${ }^{a}$ Error bars represent standard deviation for $n \geq 3$ separate syntheses.

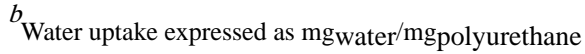

$c_{\text {Maximum instantaneous NO flux. }}$

${ }^{d}$ Half-life of NO release.

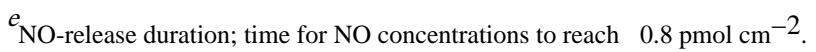

$f_{\text {Total NO release. }}$ 